\title{
Characteristics of Weight Function in a Steady-state Diffusion Optical Tomography
}

\author{
Kouichi Nitta Non-member (Kobe univ., nitta@kobe-u.ac.jp) \\ Osamu Matoba Non-member (Kobe univ., matoba@kobe-u.ac.jp) \\ Takeaki Yoshimura Member (Kobe univ. csyoshi@kobe-u.ac.jp)
}

Keywords : biological measurement, diffusion optical tomography, weight function model, optical path length

A diffusion optical tomography (DOT) is one of the noninvasive methods for biological measurements by means of near-infrared light. Figure 1 shows a schematic diagram of it. DOT is composed of an equipment for optical measurement and signal reconstruction for tomographic imaging. In this paper, a practical weight function model for signal processing in the DOT is studied.

In this research, we derive a simple model which is formed by a product of a sensitivity function of the voxel and a mean optical

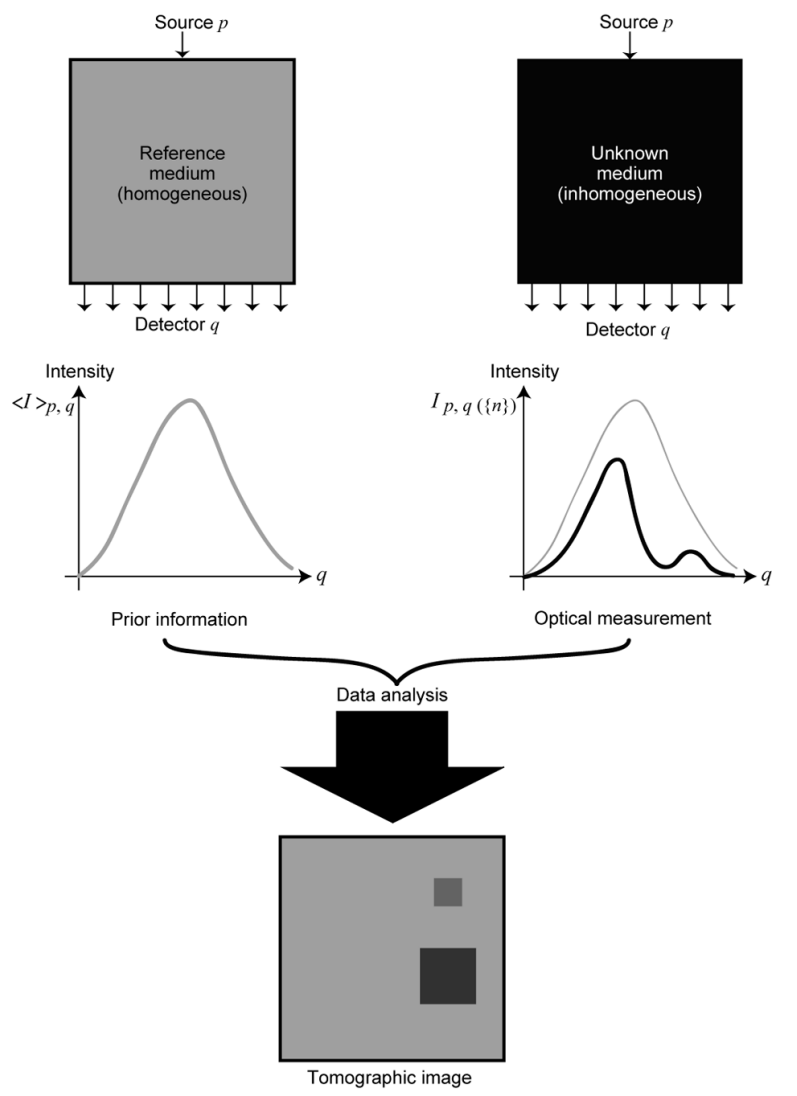

Fig. 1. Schematic diagram of a steady state diffusion optical tomography

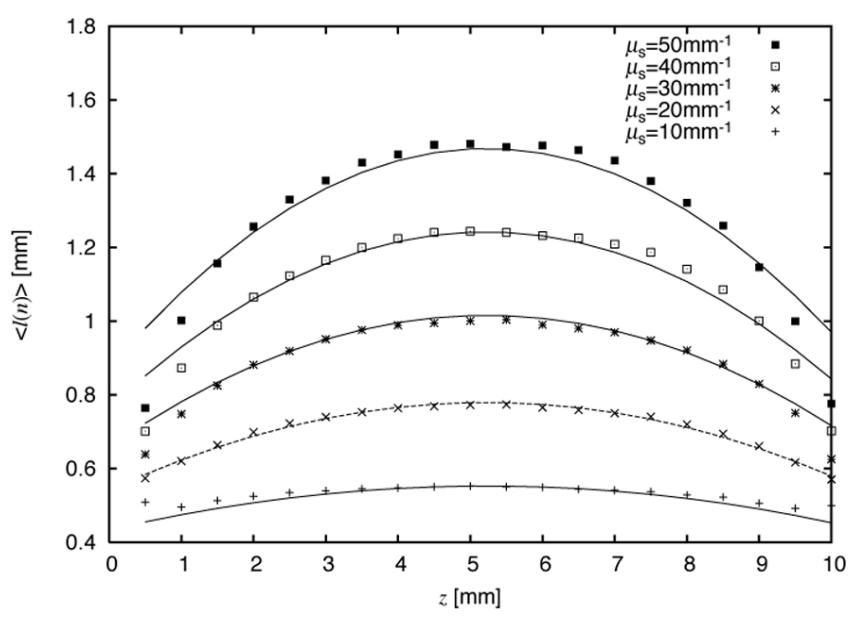

Fig. 2. Variation of the mean path-length along y-axis at $\mathrm{x}=0 \mathrm{~mm}$

path length in the voxel. The sensitivity function is defined as the intensity rate of the light passing through the voxel to the output light. The mean optical path-length is the averaged path-length of the light propagating through the voxel. The feature of the present model is that a sensitivity function and a mean optical path length are determined independently.

For analytical derivation of mean optical path length at any voxel, we invastigate on the relation between a voxel position and the path length. The analysis shows that the path length can be inferred from the optical scattering coefficient in a target medium. Also, the path length has a constant value which is independent of the source-detector arrangement. On the other hand, as shown in Fig. 2, it obeys a certain convex function depended on the average scattering coefficient in a depth direction.

It is verified that the weight function derived with the proposed model is in good agreement with the exact solution given by Monte Carlo method. The set of weight function in a target tissue can be obtained by calculating the sensitivity function and mean path-length using the mean scattering coefficient of a target tissue. Therefore, the proposed method is considered to be effective to make a DOT system practical use. 


\title{
Characteristics of Weight Function in a Steady-state Diffusion Optical Tomography
}

\author{
Kouichi Nitta* \\ Non-member \\ Osamu Matoba* \\ Non-member \\ Takeaki Yoshimura* Member
}

\begin{abstract}
For practical use of image reconstruction in a steady-state diffusion optical tomography, a weight function is analytically derived in a linear perturbation approach. We show that the weight function at any voxel in the medium is given by a product of a sensitivity function at the voxel and a mean optical pathlength in the voxel, whose two parameters have independent behavior to each other. By use of Monte Carlo simulation, it is found that the mean path length is easily obtained without stochastic calculation by scattering coefficient in the medium. Weight function derived by the proposed method is in good agreement with exact solution based on Monte Carlo method.
\end{abstract}

Keywords : biological measurement, diffusion optical tomography, weight function model, optical path length

\section{Introduction}

A technique for optical measurement with near-infrared light source has a potentiality to provide us useful information in biological tissues. The light of the wavelength region has high transmittance for the biological tissues and does not damage target media. Also, such an optical technique is useful to construct simple and compact systems with low costs.

As one of examples with an optical fiber, an optical coherence tomography (OCT) with optical interference has been presented ${ }^{(1)}$. The fiber-based OCT provides us tomographic images with optically high stability and accuracy. Post signal processing is remarkably simple because a reconstruction tecqunique such as resolving inversion problems does not be required for the tomographic imaging. As a result, OCT is applied to some practical targets in medical science such as eyeground, human skin, and so on ${ }^{(2)(3)}$. In OCT, the signal component is ballistic light which gives a reflectance information in interaction with the medium, and the noise component is caused by scattered light (diffuse light). Biological tissues are highly scattering media even when the near-infrared light is used. So, the attenuation of the signal light and the increase of the noise light due to propagation in the tissue prevent us from obtaining information on deep parts of target media. Generally, axial range to be measured with the OCT seems to be only about $1 \mathrm{~mm}$ in biological tissues. The restriction on the axial range gives a serious difficulty to medical appication of the OCT technique.

On the other hand, there is a challenging technique that the desired absorption-information is deduced by transmittance or reflectance of the diffuse light, for example, near-infrared spectroscopy ${ }^{(4)-(6)}$. In the method, firstly, optical light emitted from the fiber probe is incident to highly scattered media. The light is multiply scattered in the media. And optical intensity arriving at a detector probe is measured. The optical absorption in target media can be obtained by the measured results with various wavelength.

\footnotetext{
* Department of Computer and Systems Engineering, Faculty of Engineering, Kobe University

1-1, Rokkodaicho, Nada-ku, Kobe 657-8501
}

The characteristics may provide useful information in medical science such a density of hemoglobin, glucose, water, fat, and so on. In this technique, various researches are reported. For example, optical probe geometry is analyzed in terms of optical path length in the media ${ }^{(4)-(6)}$. And some practical systems of near-infrared spectroscopy have been developed, typically called an optical topography ${ }^{(7)}$. The technique, however, aquires the absorption information between source- and detector-probes placed on the target surface, whose information is non-resolved in the depth direction.

For depth-resolved improvements of the technique, diffusion optical tomography (DOT) is investigated although it is difficult to apply the technique to living tissues. If the tomographic imaging of the absorption information localized in highly scattering tissues can be realized with sufficient 3D-resolution, the technique will provide important functions referred with life-activities in human body ${ }^{(8)(9)}$. Moreover, biological measurement-range may be able to be achieved to the depth of several centimeters because scattered light contributes to imaging.

Generally, a DOT system consists of an optical hardware for data acquisitions and digital signal processing for image reconstruction. The optical hardware provides the data sets with multi-source as input and multi-detector as output. In the data acquisitions, a steady-state intensity, a time-domain or a frequency-domain system has been proposed. In the image reconstruction, we should utilize both the forward problem for light propagation in the tissues and the inverse one to reconstruct the absorption information, and many techniques have been proposed $^{(10)-(13)}$.

In DOT, the reliability of the reconstructed results are usually limited by many factors such as qualities of the measurement data and inaccuracies of the weight function describing light propagation in a medium. Among the various approaches proposed for image recovery, the steady-state intensity system may be expected to collect high quality data of a good signal-to-noise ratio for the required penetration depth ${ }^{(10)-(11)}$. Generally, the calculation of the weight function takes a lot of time 
by stochastic techniques such as a Monte Carlo method. For the practical application, the fast derivation of the weight function is one of important issues. In this paper, we simplify the derivation of the weight function in the steady-state tomography technique.

\section{A Weight Function for Steady-state DOT}

Figure 1 shows a schematic diagram of the stady-state DOT. Let us deduce a spatial distribution of absorption coefficients in scattering medium under the condition that unknown medium (inhomogeneous medium) to be analyzed and the corresponding reference medium (homogeneous medium) are given. In basic research, we assume that the scattering coefficient distributes spatially uniform in each medium and the coefficients in the two media are the same value. Light illuminated from a source point on the surface propagates the medium under scattering and absorption phenomenon. The modulated light is measured by photo-detectors on the other surface. The detected intensity is different between the two media. The difference depends on the spatial distribution of optical absorption in the inhomogeneous medium. Therefore, intensity-difference distributions for a given source will provide the absorption distribution of the unknown medium by resolving the inverse problem with a weight function. To prevent ill-posed problem, a large number of data-sets is acquired by means of changing the source positions and measuring the output intensity distribution every the changing.

In our method, an inhomogeneous medium containing targets is divided into small voxels. The absorption coefficient in $n$-th voxel is written by Eq. (1).

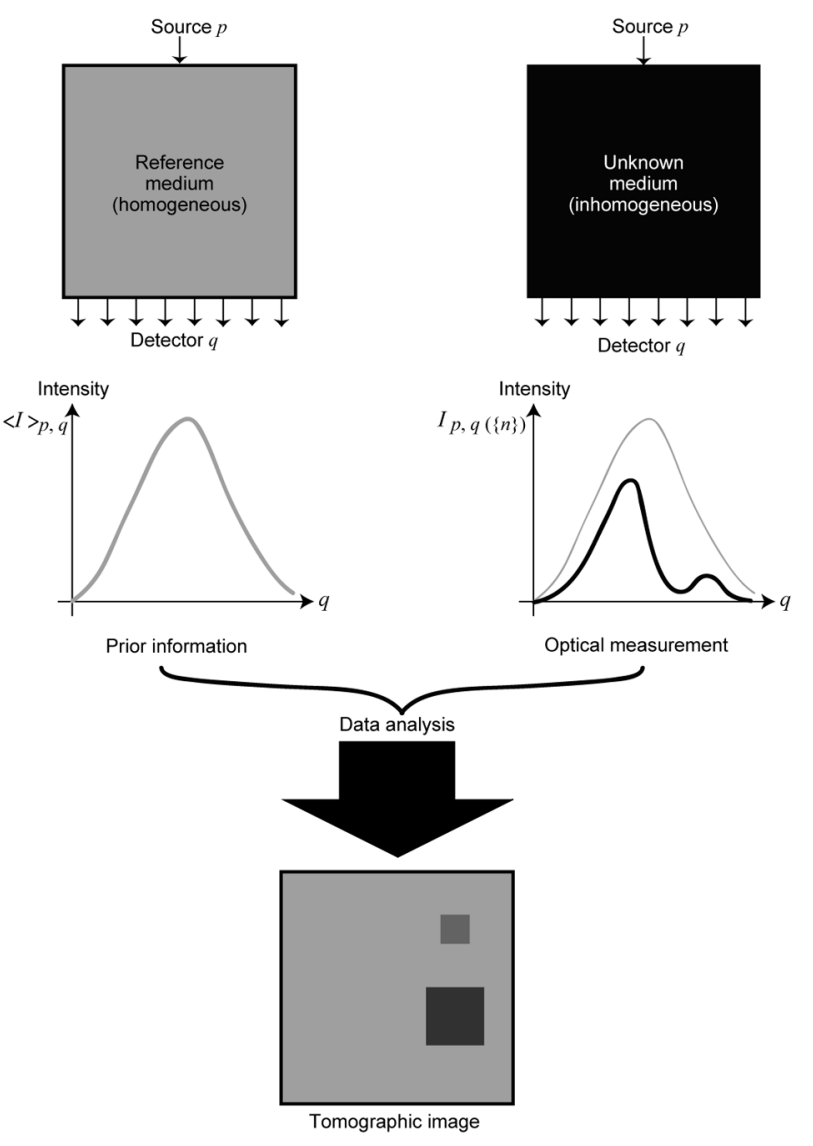

Fig. 1. Schematic diagram of a stady state diffusion optical tomography

$$
\mu_{a}(n)=<\mu_{a}>+\Delta \mu_{a}(n)
$$

In Eq. (1), $<\mu_{a}>$ and $\Delta \mu_{a}(n)$ are an average absorption coefficient and its amount of absorption change, respectively. The scattering coefficient is assumed to be a constant value of $\left\langle\mu_{s}>\right.$ through the medium.

Let us consider a pair of source voxel $p$ and detector voxel $q$ which are adjacent to surface of the medium. We assume that the output intensity be $I_{p q}(\{n\})$ where the symbol $\{n\}$ is a set through whole medium, because the absorption changes are distributed over all voxels for the inhomogeneous medium. Then, $I_{p q}(\{n\})$ represents the output intensity arriving at a detector $q$ through the real medium when total input intensity $I_{0}$ is injected into a source $p$. The light propagation in the inhomogeneous medium is described by means of a linear perturbation approach under the Born approximation. When $\langle I\rangle_{p q}$ is the output intensity for the homogeneous reference-medium, a linear perturbation equation ${ }^{(14)}$ is given by Eq. (2).

$$
\ln \left(\frac{<I>_{p q}}{I_{p q}(\{n\})}\right)=\sum_{n=1}^{N} W_{p q}(n) \Delta \mu_{a}(n)
$$

Here, $N$ is the total voxel number of whole medium and $W_{p q}(n)$ is the weight function at $n$-th voxel under the $p$ - $q$ source-detector pair. The equation is used by many researchers ${ }^{(13)}$. When the deviation $\Delta \mu_{a}(n)$ is sufficiently small, the weight function, i.e. Jacobian operator, is defined by Eq. (3) ${ }^{(15)}$

$$
W_{p q}(n)=-\frac{\partial \ln I_{p q}(n)}{\partial \mu_{a}(n)}=\frac{1}{\Delta \mu_{a}(n)} \frac{\langle I\rangle_{p q}-I_{p q}(n)}{I_{p q}(n)} .
$$

In this equation, $I_{p q}(n)$ is the output intensity for the medium with the deviation $\Delta \mu_{a}(n)$ only in $n$-th voxel.

If $\langle I\rangle_{p q}$ and $W_{p q}(n)$ are obtained as priori information, spatially distributed absorption deviation in the medium, $\Delta \mu_{a}(n)$, is calculated by Eq. (2) after $I_{p q}(\{n\})$ is measured. Such a reconstruction is well-known as a steady-state technique because only output intensity data is utilized. Ueda et al. has shown that $\langle I\rangle_{p q}$ can be obtained experimentally by so-called average value $\operatorname{method}^{(16)}$. Therefore, it is most important in the steady-state method that the weight function for the medium can be determined in advance. Generally, weight function is calculated by stochastic methods such as Monte Carlo technique. These methods take time consuming, and then it is not practical use for unknown scattering medium.

We present a simple method to obtain the weight function. Because photon-migration in a scattering medium is considered to be under a stochastic process, the probability of photon arrived at $q$-th voxel as output from $p$-th voxel as input is defined by $h_{p q}$ under the source-detector pair. Then, as shown in Fig. $2, h_{p q}$ is decomposed into two probabilities $h_{p n q}$ and $h_{p o q}$, which are whether the photon passes through $n$-th voxel or not;

$$
h_{p q}=h_{p n q}+h_{p o q} \text {. }
$$

The same manner may define the probabilities $h_{p n}$ and $h_{n q}$ where $h_{p n}$ is the probability of photon propagation from $p$-th voxel in the input to $n$-th voxel inside the medium and $h_{n q}$ is the probability of light propagation from $n$-th voxel inside the medium to $q$-th voxel at the output. Moreover, let us define the probability density function $P(n ; l)$ that the photon propagates through $n$-th voxel has an optical path length $l$ within the voxel. The probability $h_{p n q}$ of the photon propagation through $n$-th voxel in 
$p-q$ source-detector pair is described by considering photon absorption;

$$
h_{p n q}=h_{p n}\left[\int_{0}^{\infty} \exp \left[-\Delta \mu_{a}(n) l\right] P(n ; l) d l\right] h_{n q}
$$

When $I_{0}$ is the total input intensity, the output intensity in $p-q$ source-detector pair, $I_{p q}(\{n\})$, is given by Eq. (6).

$$
I_{p q}(\{n\})=I_{0}\left(h_{p o q}+h_{p n} \int_{0}^{\infty} P(n ; l) \exp \left[-\Delta \mu_{a}(n) l\right] d l h_{n q}\right)
$$

As the same consideration to homogeneous reference-medium, the output intensity is given by Eq. (7),

$$
<I>_{p q}=I_{0}\left(h_{p o q}+h_{p n} \int_{0}^{\infty} P(n ; l) d l h_{n q}\right)
$$

because $\Delta \mu_{a}(n)=0$. If Eqs. (6) and (7) are substituted into Eq. (3) and a mathematical approximation of $1-\exp \left[-\Delta \mu_{a}(n) l\right]=\Delta \mu_{a}(n) l$ is used for a small value of $\Delta \mu_{a}(n)$, then the weight function is rewritten as Eq. (8).

$$
W_{p q}(n)=S_{p q}(n)<l(n)>
$$

$S_{p q}(n)$ and $<l(n)>$ described in Eq. (8) are represented as Eqs. (9) and (10), respectively.

$$
\begin{aligned}
& S_{p q}(n)=\frac{h_{p n} h_{n q}}{h_{p q}} \ldots \ldots \ldots . . . . . . . \\
& <l(n)>=\int_{0}^{\infty} l P(n ; l) d l
\end{aligned}
$$

Here $S_{p q}(n)$ is the sensitivity function which is the intensity rate of light passing through $n$-th voxel to the output intensity under the $p-q$ source-detector pair. The $S_{p q}(n)$ can be obtained by solving the diffusion equation under the boundary condition, if the optical parameters $\left\langle\mu_{a}\right\rangle$ and $\left\langle\mu_{s}>\right.$ of homogeneous medium are given $^{(17)}$. The other parameter $<l(n)>$ is a mean path-length of photon passing through $n$-th voxel. Note that the value does not depend on the source-detector positions and moreover, in the isotropic scattering region, may be independent of $n$-th voxel position. From these considerations, Eq. (8) may be a useful formula of the weight function, that is, a product of the sensitivity function and the mean path-length in any voxels. If the mean

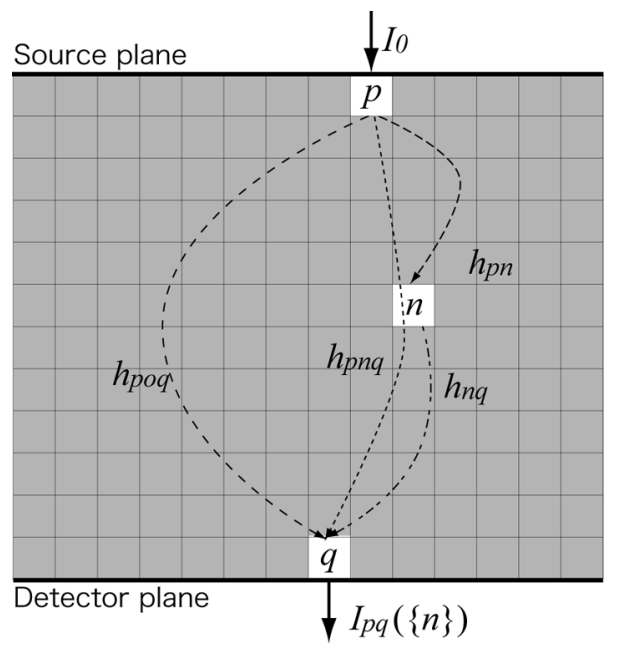

Fig. 2. Model of scattering medium path-length $<l(\mathrm{n})>$ is calculated simply, the weight function is obtained enough for practical use.

\section{Numrical Analysis of Optical Path Length in a Voxel}

We investigate a position-dependence of the mean path-length by means of Monte Carlo simulation for a thick slab model shown in Fig. 3. For simplicity, a two-dimensional medium with weak absorption and strong scattering is employed. Such a two-dimensional approach is sufficient for analyzing and demonstrating mathematical features of the photon propagation problem ${ }^{(18)(19)}$. The medium size is $128(\mathrm{~mm}) \times 10(\mathrm{~mm})$, which corresponds to $256 \times 20$ voxels. In the Fig. $3, x$ - and $z$-axes show horizontal and vertical positions of voxels, respectively. The optical parameters of the medium are set to $\left\langle\mu_{a}\right\rangle=0.01 \mathrm{~mm}^{-1}$, $<\mu_{s}>=5 \sim 50 \mathrm{~mm}^{-1}$ and the anisotropy factor of $g=0.9$. The photon is normally incident to $p$-th voxel located at a center on the surface (i.e., $x=z=0 \mathrm{~mm}$ ), and the detector voxel is located at $x=$ $0 \mathrm{~mm}$ on the other side of the surface $(z=10 \mathrm{~mm})$.

Figure 4 shows the mean path-length along the $x$-axis at the two different depths; $z=$ (a) 2.5 and (b) $5.0 \mathrm{~mm}$. For the $x$-axis, only central parts of the model are extracted on the graph. Here the fluctuation of each curve on both side of the $x$-axis is a statistical noise due to finite Monte Carlo calculations of $10^{7}$ times. For average scattering coefficients over wide range, the mean path-length is constant along the $x$ direction at each depth. The fact also shows that the mean path-length or $P(n ; l)$ as defined by Eq. (5) does not depend on the source-detector arrangement.

Figure 5 shows the depth-dependence of the mean path-length along the $z$ direction. The variation suggests to be yielded by the anisotropic scattering influence due to the surface boundary so that a photon propagated to outside of the medium could not return to the medium because there is no scattering. In this figure, one dashed curve is a convex profile function, which is fitted to the markers, derived from numerical calculations under $\left\langle\mu_{s}\right\rangle=20$ $\mathrm{mm}^{-1}$, and others solid curves are scaled by multiplying some factors to the fitted convex function (dashed curve) where the factors depend linearly on the average scattering coefficient. Each marker of calculated data coincides well with the corresponding solid curve except for near the surface yielding a strong anisotropy effect. Therefore, it is found that the mean path-length has the same convex profile functions along the $z$-direction as the medium with different average scattering coefficient. The similar character

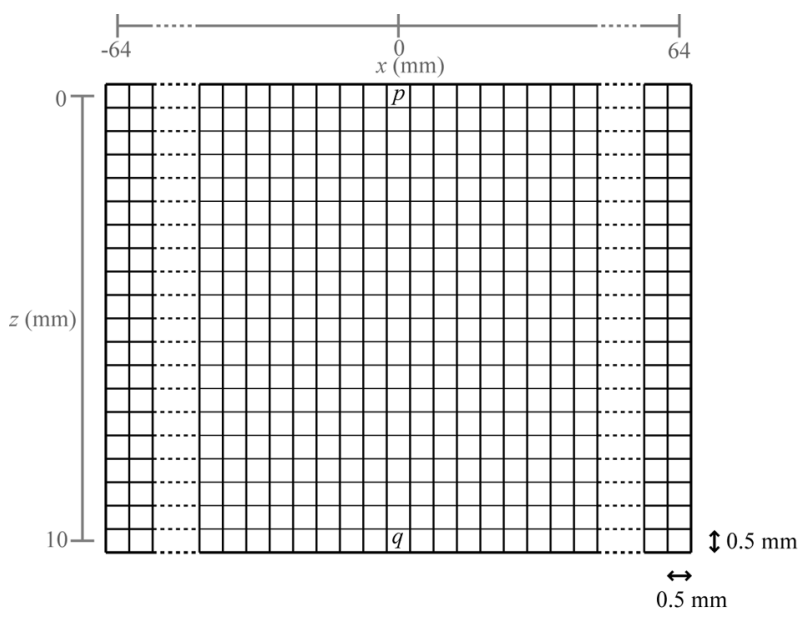

Fig. 3. A slab model for numerical analysis 


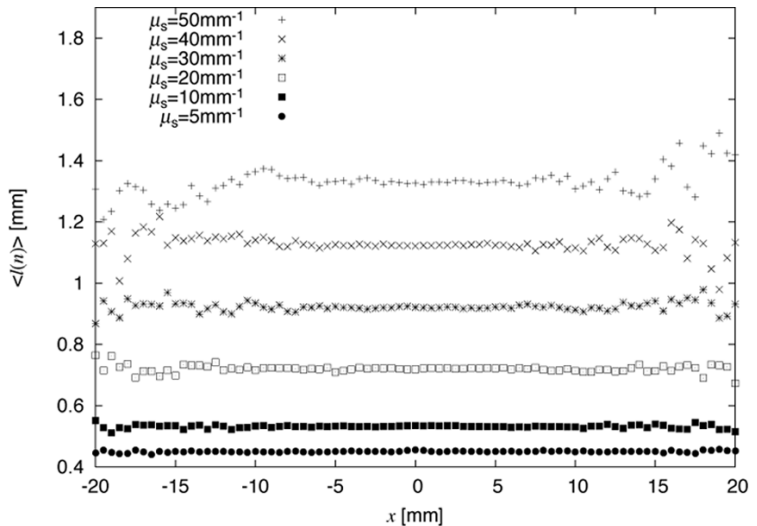

(a)

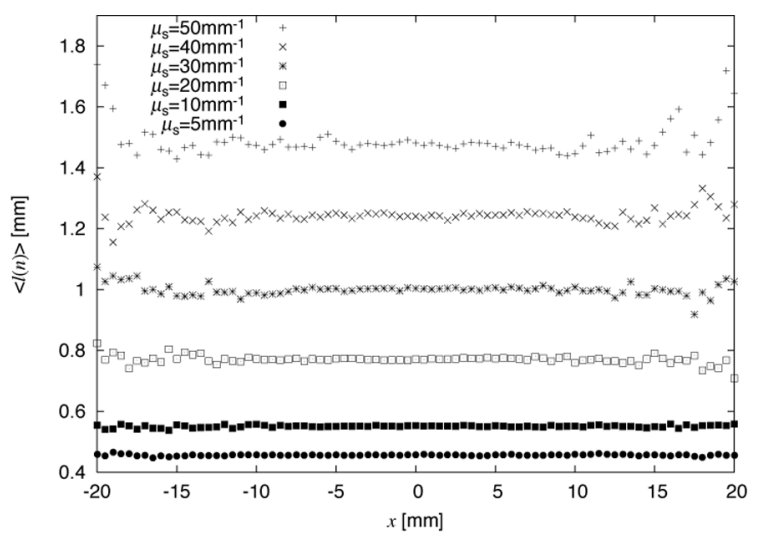

(b)

Fig. 4. Variation of the mean path-length along x-direction as a function of average scattering coefficient at the depth; (a) $z=2.5 \mathrm{~mm}$ and (b) $z=5.0 \mathrm{~mm}$; The source and detector positions are $p ;(x=z=0 \mathrm{~mm})$ and $q ;(x=0 \mathrm{~mm}, z=10 \mathrm{~mm})$

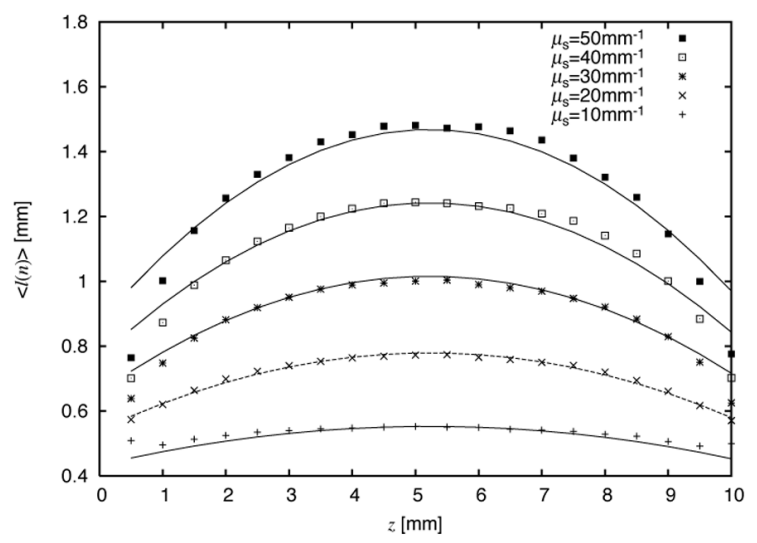

Fig. 5. Variation of the mean path-length along $\mathrm{z}$-axis at $\mathrm{x}=0$ $\mathrm{mm}$; The source and detector positions are $p ;(x=z=0 \mathrm{~mm})$ and $q ;(x=0 \mathrm{~mm}, z=10 \mathrm{~mm})$

has been also obtained even for variations of the medium thickness smaller than $20 \mathrm{~mm}$, and variation of the average absorption coefficient $\left\langle\mu_{a}\right\rangle \leq 0.1 \mathrm{~mm}^{-1}$. The characteristics about thickness range show effectiveness of the present model even though only two-dimensional model is used in our analysis. This is because the range is more ten times deeper than that in a OCT. These results suggest that the weight function of the medium with a weak absorption can be predicted from the average optical parameter of $\left\langle\mu_{s}>\right.$.

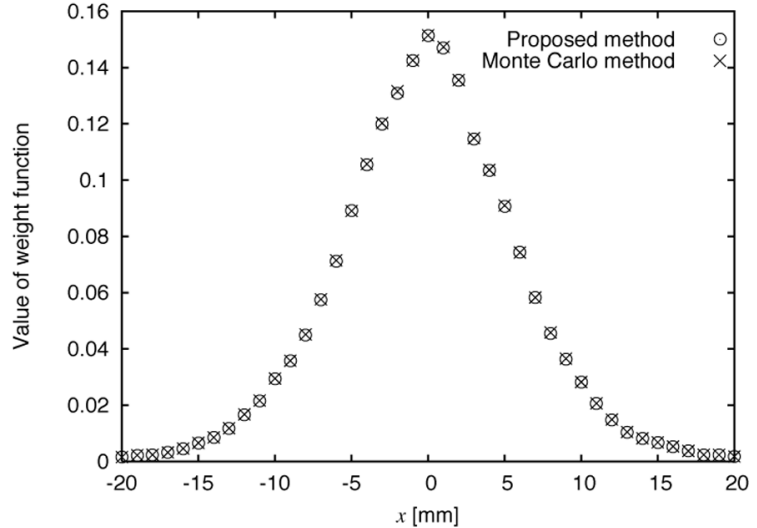

(a)

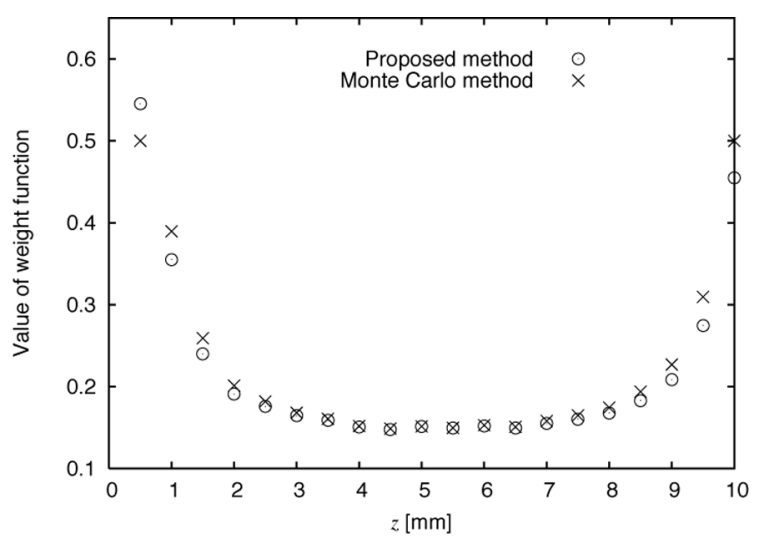

(b)

Fig. 6. Weight functions at (a) $\mathrm{z}=5.0 \mathrm{~mm}$ and (b) $\mathrm{x}=0 \mathrm{~mm}$ which are deduced by proposed method and Monte Carlo method for medium of $\left\langle\mu_{a}\right\rangle=0.01 \mathrm{~mm}^{-1}$ and $\left\langle\mu_{s}\right\rangle=10 \mathrm{~mm}^{-1}$; The source and detector positions are $p ;(x=z=0 \mathrm{~mm})$ and $q$; $(x=0 \mathrm{~mm}, z=10 \mathrm{~mm})$

We compare the weight functions of Eqs. (3) and (8) where the weight function of Eq. (3) and the sensitivity function of Eq. (9) are calculated by Monte Carlo method. Figure 6 shows the weight function obtained by Eq. (8) is in good agreement with the exact solution by Monte Carlo method. Therefore, the proposed calculation technique is useful for practical applications in DOT.

\section{Conclusion}

In this paper, we have found that the weight function of any voxel in the medium is described by a product of the sensitivity function and the mean path-length for the voxel in the linear perturbation approach. The sensitivity function is the intensity rate of the light passing through the voxel to the output light. The mean path-length is the statistically averaged path-length of the light propagating through the voxel, which has a constant value independent of the source-detector arrangement. And, in a depth direction, it obeys a certain convex function depended on both the average scattering coefficient and the medium thickness. When the mean scattering coefficient is given, weight function is obtained by calculating the sensitivity function and mean path-length without stochastic calculation. The proposed method leads to practical applications by use of DOT.

(Manuscript received July 25, 2006, revised Dec. 5, 2006) 


\section{References}

(1) D. Huang, E. A. Swanson, C. P. Lin, J. S. Shuman, W. G. Stinson, W. Chang, M. R. Hee, T. Flotte, K. Gregory, C. A. Puliafito, and J. G. Fujimoto : Science, 254, pp.1178-1181 (1991)

(2) D. C. Fernndez, H. M. Salinas, and C. A. Puliafito : Opt. Exp., Vol.13, pp.10200-10216 (2005)

(3) N. Nishizawa, Y. Chen, P. Hsiung, E. P. Ippen, and J. G. Fujimoto : Opt. Lett., Vol.29, pp.2846-2848 (2004)

(4) G. Kumar and J. M. Schmitt : Appl. Opt., Vol.36, pp.2286-2293 (1997)

(5) J. R. Mourant, I. J. Bigio, D. A. Jack, T. M. Johnson, and H. D. Miller : Appl. Opt., Vol.36, pp.5655-5661 (1997)

(6) H. Liu : Appl. Opt., Vol.40, pp.1742-1746 (2001)

(7) A. Maki, Y. Yamashita, Y. Ito, E. Watanabe, Y. Mayanagi, and H. Koizumi : Medical Phys., Vol.22, pp.1997-2005 (1995)

(8) A. Y. Bluestone, G. Abdoulaev, C. H. Schmitz, R. L. Barbour, and A. H. Hielscher : Opt. Exp. Vol.9, pp.272-286 (2001)

(9) H. Dehghani and D. T. Delpy : Appl. Opt. Vol.39, pp.4721-4729 (2000)

(10) B. W. Pogue, T. O. McBride, J. Prewitt, U. L. Osterberg, and K. D. Paulsen : Appl. Opt., Vol.38, pp.2950-2961 (1999)

(11) Y. Tsuchiya : J. Opt. Soc. Am. A, Vol.19, pp.1383-1389 (2002)

(12) B. W. Pogue, S. Geimer, T. O. McBride, S. Jiang, U. L. Osterberg, and K. D. Paulsen : Appl. Opt., Vol.40, pp.588-600 (2001)

(13) Y. Pei, H. L. Graber, and R. L. Barbour : Appl. Opt., Vol.40, pp.5755-5769 (2001)

(14) H. L. Graber, J. Chang, R. Aronson, and R. L. Barbou : Proc. of SPIE, IS11, pp.121-143 (1993)

(15) H. L. Graber, J. Chang, J. Lubowsky, R. Aronson, and R. L. Barbou : Proc. of SPIE, 1888, pp.372-386 (1993)

(16) Y. Ueda, K. Ohta, M. Oda, M. Miwa, Y. Tsuchiya, and Y. Yamashita : Appl. Opt., Vol.40, pp.6349-6355 (2001)

(17) Z. Wang, G. Y. Panasyuk, V. A. Markel, and J. C. Schotland : Opt. Lett., Vol.30, pp.3338-3340 (2005)

(18) Y. P. Kumar and R. M. Vasu : J. Biomed. Opt., pp.1002-1012 (2004)

(19) A. Sassaroli, C. Blumetti, F. Martelli, L. Alianelli, D. Contini, A. Ismaelli, and G. Zaccanti : Appl. Opt., Vol.37, pp.7392-7400 (1998)

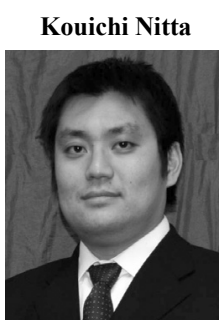

(Non-member) was born in Shiga, Japan born in Shiga Japan, on March 13, 1975. He receives the M. E. and Ph. D from Osaka University, Japan, in 2000 and 2003, respectively. He is now a research associate in Kobe University, Japan. His research interest are in optical signal processing, large scale parallel processing, image processing, and otpical measurement. $\mathrm{He}$ is a member of JSAP, OSJ, IEEE/LEOS, and SPIE.

Osamu Matoba

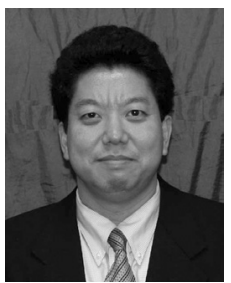

Takeaki Yoshimura

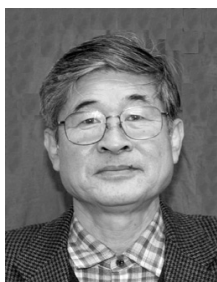

(Non-member) received the Dr. Eng. degree from Osaka University in 1996. Dr. Matoba is an associate professor in Kobe University since 2002. His current research interests include holographic data storage, optical security, and three-dimensional image processing based on digital holography. He is a member of JSAP, OSJ, OSA, IEEE, and SPIE.

Member) was born in Fukui, Japan, on March, 1944. He received the M. E. and D. E from Osaka University, Japan, in 1968 and 1972, respectively. He is now a professor in Kobe University, Japan. His research interest are in applied optics, especially optical sensing, image processing. 